\title{
Tranexamic acid reduces intraoperative blood loss in orthognathic surgery
}

\section{Abstracted from \\ Song G, Yang P, Hu J, Zhu S, Li Y, Wang Q.}

The effect of tranexamic acid on blood loss in orthognathic surgery: a meta-analysis of randomized controlled trials. Oral Surg Oral Med Oral Pathol Oral Radiol 2013; 115: 595-600. doi: 10.1016/j.oooo.2012.09.085. Epub 2012 Dec 20. Review. PubMed PMID: 23260768.

\section{Question: Does tranexamic acid reduce blood loss during orthognathic surgery?}

Data sources Medline, Embase and the reference lists of identified articles were also scanned for relevant papers.

Study selection Only randomised controlled trials in English were considered.

Data extraction and synthesis Two reviewers extracted data independently and study quality was assessed using the Jadad score. Meta-analysis was conducted.

Results Four RCTs with a total of 183 patients were included. Intraoperative blood loss in the tranexamic acid group was statistically reduced (weighted mean difference $[\mathrm{WMD}]=-93.56,95 \% \mathrm{Cl}=-132.59$ $54.52, \mathrm{P}<.00001)$. No statistical difference was seen in the postoperative levels of haemoglobin and haematocrit $(\mathrm{WMD}=0.50,95 \% \mathrm{Cls}=$ $-0.43-1.43, \mathrm{P}=.29$ and $\mathrm{WMD}=0.18,95 \% \mathrm{Cls}=-1.64-1.99, \mathrm{P}=.85$, respectively).

Conclusions This meta-analysis confirms that tranexamic acid can effectively reduce intraoperative blood loss in orthognathic surgery, especially by intravenous administration. But tranexamic acid cannot affect postoperative levels of haemoglobin and haematocrit.

\section{Commentary}

Reducing blood loss during minor and major surgical procedures to minimise complications and the need for a blood transfusion and its associated cost, and concerns for the safety related to the procedure, is an appropriate topic for the medical and dental professions. The use of anti-fibrinolytic drugs is commonly approved towards that objective. The concern is that some of the drugs used for that purpose could be associated with severe adverse effects such as vascular occlusion, stroke, increased risk of cardiovascular complications, renal dysfunction and death. ${ }^{1}$

Tranexamic acid (TXA) is one of the anti-fribrinolytic drugs used commonly for that purpose. The efficacy in reducing bleeding compared to a placebo in the case of orthognathic surgery is the topic selected in this review.

The authors searched two databases and hand searched for randomised clinical trials in English only. The four articles that were selected for final inclusion contained the use of tranexamic acid compared to a placebo. The authors used the JADAD scale of five points to critically appraise the quality of the randomised clinical trials and assigned the studies a high score according to the scale (mean score $>3$ ).

The combination of the data through the meta-analysis confirmed the effectiveness of tranexamic acid as a viable treatment to prevent blood loss during orthognathic surgery. In regard to the intraoperative blood loss, the studies showed that tranexamic acid had the capability of reducing intraoperative blood loss compared with the placebo groups.

Although the results varied based on the administered route the results obtained from intravenous administration were statistically significant. Topical application of TXA was not statistically significant. Those studies that reported the pre- and post-operative haemoglobin and haematocrit level showed that the changes were not statistically significant.

However, the authors recommended that the results of the metaanalysis should be interpreted with caution due to some bias from the studies and the low number of participants in each study. They also noted that the professionals should also consider that the type of orthognathic surgery might influence the volume of blood loss and that tranexamic acid via intravenous administration appears to be more effective.

Even though the results are statistically significant, favouring the intervention, the confidence interval is wide and reduces the precision of the results.

A very thorough Cochrane systematic review published in 2011 to examine the evidence for the efficacy of antifibrinolytic drugs, which included tranexamic acid, in reducing blood transfusions and any effect on clinical outcomes, included 65 randomised clinical trials (4842 patients in total). It compared the use of tranexamic acid in a wide variety of surgeries including cardiac surgery to demonstrate the efficacy of TXA.

The side effects were also analysed and were found to be minimal. The risk of cerebro-vascular accidents and other side effects were not associated with the use of intravenous TXA.

The conclusions of this review confirmed what the Cochrane review had concluded prior, with regard to major surgeries.

Camelia Espahbod and Analia Veitz-Keenan NYU College of Dentistry, New York, USA

\footnotetext{
1. Henry DA, Carless PA, Moxey AJ, et al. Anti-fibrinolytic use for minimising perioperative allogeneic blood transfusion. Cochrane Database Syst Rev 2011; 3: Art. No: CD001886. DOI: 10.1002/14651858.CD001886.pub4.

Evidence-Based Dentistry (2014), 15 63. doi: 10.1038/sj.ebd6401033
} 